\title{
SOME RESULTS ON THE SUBADDITIVITY CONDITION OF SYZYGIES
}

\author{
ABED ABEDELFATAH
}

\begin{abstract}
Let $S=K\left[x_{1}, \ldots, x_{n}\right]$, where $K$ is a field, and $t_{i}$ denotes the maximal shift in the minimal graded free $S$-resolution of the graded algebra $S / I$ at degree $i$. In this paper, we prove:
\end{abstract}

- If $I$ is a monomial ideal of $S$ and $a \geq b-1 \geq 0$ are integers such that $a+b \leq \operatorname{proj} \operatorname{dim}(S / I)$, then

$$
t_{a+b} \leq t_{a}+t_{1}+t_{2}+\cdots+t_{b}-\frac{b(b-1)}{2} .
$$

- If $I=I_{\Delta}$ where $\Delta$ is a simplicial complex such that $\operatorname{dim}(\Delta)<t_{a}-a$ or $\operatorname{dim}(\Delta)<t_{b}-b$, then

$$
t_{a+b} \leq t_{a}+t_{b}
$$

- If $I$ is a monomial ideal that minimally generated by $m_{1}, \ldots, m_{r}$ such that $\frac{\operatorname{lcm}\left(m_{1}, \ldots, m_{r}\right)}{\operatorname{lcm}\left(m_{1}, \ldots, \widehat{m}_{i}, \ldots, m_{r}\right)} \notin K$ for all $i$, where $\widehat{m}_{i}$ means that $m_{i}$ is omitted, then $t_{a+b} \leq t_{a}+t_{b}$ for all $a, b \geq 0$ with $a+b \leq$ $\operatorname{proj} \operatorname{dim}(S / I)$.

\section{INTRODUCTION}

Let $S=K\left[x_{1}, \ldots, x_{n}\right]$, where $K$ is a field and let $I$ be a graded ideal of $S$ and suppose $S / I$ has minimal graded free $S$-resolution

$0 \rightarrow F_{p}=\bigoplus_{j \in \mathbb{N}} S(-j)^{\beta_{p, j}} \rightarrow \cdots \rightarrow F_{1}=\bigoplus_{j \in \mathbb{N}} S(-j)^{\beta_{1, j}} \rightarrow F_{0}=S \rightarrow S / I \rightarrow 0$.

The numbers $\beta_{i, j}=\beta_{i, j}(S / I)$, where $i, j \geq 0$, are called the graded Betti numbers of $I$, which count the elements of degree $j$ in a minimal generator of $(i+1)$-th syzygy: $\operatorname{Syz}_{i+1}(S / I)=\operatorname{ker}\left(F_{i} \rightarrow F_{i-1}\right)$. Let $t_{i}$ denote the maximal shifts in the minimal graded free $S$-resolution of $S / I$, namely

$$
t_{i}=t_{i}(S / I):=\max \left(j: \beta_{i, j}(S / I) \neq 0\right) .
$$

We say that $I$ satisfies the subadditivity condition if $t_{a+b} \leq t_{a}+t_{b}$, for all $a, b \geq 0$ and $a+b \leq p$, where $p$ is the projective dimension of $I$.

It is known that graded ideals may not satisfy the subadditivity condition as shown by the counter example in [3, Section 5.4]. However, no counter examples are known for monomial ideals. For edge ideals of graphs the inequality $t_{a+1} \leq t_{a}+t_{1}$ was shown by Fernández-Ramos and Gimenez [1,

Key words and phrases. Betti numbers, simplicial complex, monomial ideal, subadditivity condition. 
Theorem 4.1]. The same inequality has been shown later for any monomial ideal [2, Corollary 4] by Srinivasan and Herzog. Yazdan Pour independently proved the same result in [4, Corollary 3.5]. Bigdeli and Herzog proved the subadditivity condition, when $I$ is the edge ideal of chordal graph or whisker graph [8, Theorem 1]. Some more results regarding subadditivity have been obtained by Khoury and Srinivasan [5, Theorem 2.3], the author and Nevo [6, Theorem 1.3] and Faridi [7, Theorem 3.7].

In this paper we prove in Theorem 3.4 that if $I$ is a monomial ideal of $S$ and $a \geq b-1 \geq 0$ are integers such that $a+b \leq \operatorname{proj} \operatorname{dim}(S / I)$, then

$$
t_{a+b} \leq t_{a}+t_{1}+t_{2}+\cdots+t_{b}-\frac{b(b-1)}{2},
$$

which generalizes the well-known inequality $t_{a+1} \leq t_{a}+t_{1}$.

In Theorem 3.3, we prove that if $I=I_{\Delta}$ where $\Delta$ is a simplicial complex such that $\operatorname{dim}(\Delta)<t_{a}-a$ or $\operatorname{dim}(\Delta)<t_{b}-b$, then $t_{a+b} \leq t_{a}+t_{b}$. The proof of both Theorems 3.3 and 3.4 uses a combinatorial topological argument.

In Theorem 4.1, we prove algebraically, using Taylor resolution, that the subadditivity condition holds when $I$ is a monomial ideal that minimally generated by $m_{1}, \ldots, m_{r}$ such that $\frac{\operatorname{lcm}\left(m_{1}, \ldots, m_{r}\right)}{\operatorname{lcm}\left(m_{1}, \ldots, \widehat{m}_{i}, \ldots, m_{r}\right)} \notin K$ for all $i$, where $\widehat{m}_{i}$ means that $m_{i}$ is omitted.

\section{Preliminaries}

Fix a field $K$. Let $S=K\left[x_{1}, \ldots, x_{n}\right]$ be the graded polynomial ring with $\operatorname{deg}\left(x_{i}\right)=1$ for all $i$, and $M$ be a graded $S$-module. The integer $\beta_{i, j}^{S}(M)=\operatorname{dim}_{K} \operatorname{Tor}_{i}^{S}(M, K)_{j}$ is called the $(i, j)$ th graded Betti number of $M$. Note that if $I$ is a graded ideal of $S$, then $\beta_{i+1, j}^{S}(S / I)=\beta_{i, j}^{S}(I)$ for all $i, j \geq 0$.

For a simplicial complex $\Delta$ on the vertex set $\Delta_{0}=[n]=\{1, \ldots, n\}$, its Stanley-Reisner ideal $I_{\Delta} \subset S$ is the ideal generated by the squarefree monomials $x_{F}=\prod_{i \in F} x_{i}$ with $F \notin \Delta, F \subset[n]$. The dimension of the face $F$ is $|F|-1$ and the dimension of $\Delta$ is $\max \{\operatorname{dim} F: F \in \Delta\}$.

For $W \subset V$, we write

$$
\Delta[W]=\{F \in \Delta: F \subset W\}
$$

for the induced subcomplex of $\Delta$ on $W$. We denote by $\beta_{i}(\Delta)=\operatorname{dim}_{K} \widetilde{H}_{i}(\Delta ; K)$ the dimension of the $i$-th reduced homology group of $\Delta$ with coefficients in $K$. The following result is known as Hochster's formula for graded Betti numbers.

Theorem 2.1 (Hochster). Let $\Delta$ be a simplicial complex on $[n]$. Then

$$
\beta_{i, i+j}\left(S / I_{\Delta}\right)=\sum_{W \subset[n],|W|=i+j} \beta_{j-1}(\Delta[W] ; K)
$$

for all $i, j \geq 0$. 
If $\Delta_{1}$ and $\Delta_{2}$ are two subcomplexes of $\Delta$ such that $\Delta=\Delta_{1} \cup \Delta_{2}$, then there is a long exact sequence of reduced homologies, called the Mayer-Vietoris sequence

$$
\cdots \rightarrow \widetilde{H}_{i}\left(\Delta_{1} \cap \Delta_{2} ; K\right) \rightarrow \widetilde{H}_{i}\left(\Delta_{1} ; K\right) \oplus \widetilde{H}_{i}\left(\Delta_{2} ; K\right) \rightarrow \widetilde{H}_{i}(\Delta ; K) \rightarrow \widetilde{H}_{i-1}\left(\Delta_{1} \cap \Delta_{2} ; K\right) \rightarrow \cdots .
$$

\section{The MAIn Theorems}

Lemma 3.1. Let $\Delta=\Delta_{1} \cup \cdots \cup \Delta_{t}$ be a union of subcomplexes. If

$$
\widetilde{H}_{j-r+1}\left(\bigcap_{m=1}^{r} \Delta_{i_{m}} ; K\right)=0
$$

for all $1 \leq i_{1}<\cdots<i_{r} \leq t$, then $\widetilde{H}_{j}(\Delta ; K)=0$.

Proof. We prove the assertion by induction on $t$. It is trivial for $t=1$. Let $t=2$. Using the Mayer-Vietoris sequence

$$
\cdots \rightarrow \widetilde{H}_{j}\left(\Delta_{1} ; K\right) \oplus \widetilde{H}_{j}\left(\Delta_{2} ; K\right) \rightarrow \widetilde{H}_{j}(\Delta ; K) \rightarrow \widetilde{H}_{j-1}\left(\Delta_{1} \cap \Delta_{2} ; K\right) \rightarrow \cdots
$$

and the assumptions $\widetilde{H}_{j}\left(\Delta_{1} ; K\right)=\widetilde{H}_{j}\left(\Delta_{2} ; K\right)=\widetilde{H}_{j-1}\left(\Delta_{1} \cap \Delta_{2} ; K\right)=0$, we get $\widetilde{H}_{j}(\Delta ; K)=0$.

Let $t>2$. Now, we consider the Mayer-Vietoris sequence

$\cdots \rightarrow \widetilde{H}_{j}\left(\Delta_{1} ; K\right) \oplus \widetilde{H}_{j}\left(\Delta_{2} \cup \cdots \cup \Delta_{t} ; K\right) \rightarrow \widetilde{H}_{j}(\Delta ; K) \rightarrow \widetilde{H}_{j-1}\left(\left(\Delta_{1} \cap \Delta_{2}\right) \cup \cdots \cup\left(\Delta_{1} \cap \Delta_{t}\right) ; K\right) \rightarrow \cdots$

by induction assumption, we have

$$
\widetilde{H}_{j}\left(\Delta_{2} \cup \cdots \cup \Delta_{t} ; K\right)=\widetilde{H}_{j-1}\left(\left(\Delta_{1} \cap \Delta_{2}\right) \cup \cdots \cup\left(\Delta_{1} \cap \Delta_{t}\right) ; K\right)=0 .
$$

Hence $\widetilde{H}_{j}(\Delta ; K)=0$ as desired.

Proposition 3.2. Let $\Delta$ be a simplicial complex on the set $[n]$ and $W \subseteq[n]$ such that $|W|=t_{a}+s+l+1$, where $a \leq \operatorname{proj} \operatorname{dim}\left(S / I_{\Delta}\right), s \geq 0$ and $l \geq 1$. Then for all $A \subseteq W$ with $|A|=s^{\prime}+l$ where $0 \leq s^{\prime} \leq s$, we have

$$
\widetilde{H}_{t_{a}-a+s}\left(\bigcup_{B \subseteq A:|B|=l}(\Delta[W \backslash B] ; K)\right)=0 .
$$

Proof. We prove the proposition by induction on $l$. Let $l=1$. Note that for all $1 \leq r \leq|A|$, we have $\beta_{a, t_{a}+s+2-r}\left(S / I_{\Delta}\right)=0$. So

$$
\widetilde{H}_{t_{a}-a+s-r+1}\left(\Delta\left[W \backslash B_{1}\right] \cap \cdots \cap \Delta\left[W \backslash B_{r}\right] ; K\right)=0 .
$$

for all distinct singleton subsets $B_{1}, \ldots, B_{r}$ of $A$. By Lemma 3.1, we obtain that

$$
\widetilde{H}_{t_{a}-a+s}\left(\bigcup_{B \subseteq A:|B|=1}(\Delta[W \backslash B] ; K)\right)=0 .
$$


Let $l>1$ and $A=\left\{b_{1}, \ldots, b_{s^{\prime}}, b_{s^{\prime}+1}, \ldots, b_{s^{\prime}+l}\right\}$. For $1 \leq j \leq s^{\prime}+1$, denote by $B_{j}$ the set of all $B \subseteq A$ such that $|B|=l, b_{j} \in B$ and $\left\{b_{1}, \ldots, b_{j-1}\right\} \cap B=\emptyset$. For $1 \leq j \leq s^{\prime}+1$, let $\Delta_{j}=\bigcup_{B \in B_{j}} \Delta[W \backslash B]$. We have that

$$
\bigcup_{B \subseteq A:|B|=l}(\Delta[W \backslash B])=\Delta_{1} \cup \cdots \cup \Delta_{s^{\prime}+1} .
$$

Let $1 \leq i_{1}<\cdots<i_{r} \leq s^{\prime}+1$. Denote by $W^{\prime}$ the set $W \backslash\left\{b_{i_{1}}, \ldots, b_{i_{r}}\right\}$ and by $A^{\prime}$ the set $A \backslash\left\{b_{1}, \ldots, b_{i_{r}}\right\}$. Let $\bar{s}=s-r+1$ and $\bar{l}=l-1$. We have that $\left|W^{\prime}\right|=t_{a}+\bar{s}+\bar{l}+1$ and

$$
\Delta_{i_{1}} \cap \cdots \cap \Delta_{i_{r}}=\bigcup_{B \subseteq A^{\prime}:|B|=\bar{l}} \Delta\left[W^{\prime} \backslash B\right] .
$$

By induction hypothesis, we have that

$$
\widetilde{H}_{t_{a}-a+\bar{s}}\left(\Delta_{i_{1}} \cap \cdots \cap \Delta_{i_{r}} ; K\right)=0 .
$$

By Lemma 3.1 we get

$$
\widetilde{H}_{t_{a}-a+s}\left(\bigcup_{B \subseteq A:|B|=l}(\Delta[W \backslash B] ; K)\right)=0 .
$$

Now, we prove the main results.

Theorem 3.3. Let $\Delta$ be a simplicial complex on the set $[n]$ and $a, b$ are non negative integers such that $a, b \leq \operatorname{proj} \operatorname{dim}\left(S / I_{\Delta}\right)$. If $\operatorname{dim}(\Delta)<t_{a}-a$ or $\operatorname{dim}(\Delta)<t_{b}-b$, then $t_{a+b} \leq t_{a}+t_{b}$.

Proof. Without loss of generality, assume that $\operatorname{dim}(\Delta)<t_{b}-b$. We have to show that $\beta_{a+b, t_{a}+t_{b}+r+1}\left(S / I_{\Delta}\right)=0$ for all integer $r \geq 0$. Let $W \subseteq[n]$ of size $t_{a}+t_{b}+r+1$ and $A$ be any subset of $W$ with $|A|=t_{b}+r$, where $r \geq 0$. Since $\Delta$ does not contains a face of dimension $t_{b}-b$, it follows that

$$
\Delta[W]=\bigcup_{B \subseteq A:|B|=b} \Delta[W \backslash B] .
$$

By 3.2 (taking $s=s^{\prime}=t_{b}-b+r$ and $l=b$ ), it follows that

$$
\widetilde{H}_{t_{a}-a+t_{b}-b+r}(\Delta[W] ; K)=0 .
$$

Theorem 3.4. If $I$ is a monomial ideal of $S, b \geq 1$ and $a \geq b-1$ are integers such that $a+b \leq \operatorname{proj} \operatorname{dim}(S / I)$, then

$$
t_{a+b} \leq t_{a}+t_{1}+t_{2}+\cdots+t_{b}-\frac{b(b-1)}{2} .
$$

Proof. By polarization, we may assume that $I$ is a squarefree ideal, and so $I=I_{\Delta}$, where $\Delta$ is a simplicial complex on $\left[n^{\prime}\right]$. First, we prove that

$$
t_{c+1} \leq t_{c}+t_{d}-d+1,
$$


for all $d \geq 1$ and $d-1 \leq c \leq \operatorname{proj} \operatorname{dim}(S / I)$. Assume on contrary, that $\beta_{c+1, t_{c}+t_{d}-d+r+2}(S / I) \neq 0$ for some $r \geq 0$. It follows that there exists a subset $W$ of $\left[n^{\prime}\right]$ so that $|W|=t_{c}+t_{d}-d+r+2$ and $\widetilde{H}_{t_{c}-c+t_{d}-d+r}(\Delta[W] ; K) \neq$ 0 . In particular, $\operatorname{proj} \operatorname{dim}\left(S / I_{\Delta[W]}\right) \geq c+1$.

If $\operatorname{deg}(m) \geq t_{d}-d+r+2$ for all minimal generator $m$ of $I_{\Delta[W]}$, then $\beta_{1, x}\left(S / I_{\Delta[W]}\right)=0$ for all $x \leq t_{d}-d+r+1$. So $\beta_{d, x}\left(S / I_{\Delta[W]}\right)=0$ for all $x \leq t_{d}-d+r+d=t_{d}+r$. It follows that proj $\operatorname{dim}\left(S / I_{\Delta[W]}\right)<d \leq c+1$. This is a contradiction.

We obtain that there is a subset $A$ of $W$ with $|A|=t_{d}-d+r+1$ and

$$
\Delta[W]=\bigcup_{B \subseteq A:|B|=1} \Delta[W \backslash B] .
$$

By 3.2 (taking $s=s^{\prime}=t_{d}-d+r$ and $l=1$ ), it follows that

$$
\widetilde{H}_{t_{c}-c+t_{d}-d+r}(\Delta[W] ; K)=0,
$$

a contradiction.

Now, we prove by induction on $1 \leq b^{\prime} \leq b$ that

$$
t_{a+b^{\prime}} \leq t_{a}+t_{1}+t_{2}+\cdots+t_{b^{\prime}}-\frac{b^{\prime}\left(b^{\prime}-1\right)}{2} .
$$

The case $b^{\prime}=1$ follows by (1). Let $b^{\prime}>1$. By the induction hypothesis, we have

$$
\begin{aligned}
t_{a+b^{\prime}}=t_{\left(a+b^{\prime}-1\right)+1} & \leq t_{a+b^{\prime}-1}+t_{b^{\prime}}-b^{\prime}+1 \\
& \leq t_{a}+t_{1}+\cdots+t_{b^{\prime}-1}-\frac{\left(b^{\prime}-1\right)\left(b^{\prime}-2\right)}{2}+t_{b^{\prime}}-b^{\prime}+1 \\
& =t_{a}+t_{1}+t_{2}+\cdots+t_{b^{\prime}}-\frac{b^{\prime}\left(b^{\prime}-1\right)}{2} .
\end{aligned}
$$

\section{The TAYlor Resolution}

Let $I$ be a monomial ideal with $G(I)=\left\{m_{1}, \ldots, m_{r}\right\}$. For a subset $F$ of $G(I)$, set $\operatorname{lcm}(F)=\operatorname{lcm}\left\{m_{i}: m_{i} \in F\right\}$ and define a formal symbol $[F]$ with multidegree equal to $\operatorname{lcm}(F)$. Let $T_{0}=S$ and for each $i \geq 1$, let $T_{i}$ be the free $S$-module with basis $\{[F]:|F|=i\}$. Note that $T_{i}$ is a multigraded $S$-module.

Let $\phi_{0}: T_{0} \rightarrow S / I$ be the canonical homomorphism and define the multigraded differential $\phi_{i}: T_{i} \rightarrow T_{i-1}$ by

$$
[F] \longmapsto \sum_{k=1}^{i}(-1)^{k-1} \cdot \frac{\operatorname{lcm}(F)}{\operatorname{lcm}\left(F \backslash\left\{m_{j_{k}}\right\}\right)} \cdot\left[F \backslash\left\{m_{j_{k}}\right\}\right]
$$

where $F=\left\{m_{j_{1}}, \ldots, m_{j_{i}}\right\}$, written with the indices in increasing order.

The free resolution

$$
\mathbb{T}: 0 \longrightarrow T_{r} \stackrel{\phi_{r}}{\longrightarrow} \cdots \longrightarrow T_{1} \stackrel{\phi_{1}}{\longrightarrow} T_{0} \stackrel{\phi_{0}}{\longrightarrow} S / I \longrightarrow 0
$$


is called the Taylor resolution of $I$.

We denote by $H_{a}(\overline{\mathbb{T}})$ the a-th homology group, of the chain complex

$$
\overline{\mathbb{T}}: 0 \longrightarrow T_{r} \otimes_{S} K \stackrel{\phi_{r} \otimes_{S} K}{\longrightarrow} \cdots \longrightarrow T_{1} \otimes_{S} K \stackrel{\phi_{1} \otimes_{S} K}{\longrightarrow} T_{0} \otimes_{S} K \longrightarrow 0 .
$$

Note that $\beta_{a, b}(S / I)=\operatorname{dim}_{K}\left(H_{a}(\overline{\mathbb{T}})\right)_{b}$ and for all $0 \leq j \leq r$

$$
T_{j} \otimes_{S} K \cong T_{j} \otimes_{S} S / M \cong T_{j} / M T_{j}=\bar{T}_{j}
$$

where $M$ is the maximal ideal $\left(x_{1}, \ldots, x_{n}\right)$ of $S$.

Proposition 4.1. Let I be a monomial ideal with $G(I)=\left\{m_{1}, \ldots, m_{r}\right\}$. If there exists $0 \neq[F] \in H_{a+b}(\overline{\mathbb{T}})$ such that $\operatorname{deg}(\operatorname{lcm}(F))=t_{a+b}$, then $t_{a+b} \leq$ $t_{a}+t_{b}$.

Proof. With out loos of generality, assume that $F=\left\{w_{1}, \ldots, w_{a+b}\right\}$ and

$$
G(I)=\left\{m_{1}, \ldots, m_{r-(a+b)}, w_{1}, \ldots, w_{a+b}\right\} .
$$

Set $F_{1}=\left\{w_{1}, \ldots, w_{a}\right\}$ and $F_{2}=\left\{w_{a+1}, \ldots, w_{a+b}\right\}$. Since $[F] \in \operatorname{ker} \bar{\phi}_{a+b}$, it follows that $\frac{\operatorname{lcm}(F)}{\operatorname{lcm}(G)} \notin K$, for all $G \subsetneq F$. So we have that $\left[F_{1}\right] \in \operatorname{ker} \bar{\phi}_{a}$ and $\left[F_{2}\right] \in \operatorname{ker} \bar{\phi}_{b}$. If $\left[F_{1}\right] \in \operatorname{Im} \bar{\phi}_{a+1}$, then there exist $\left[F_{1,1}\right], \ldots,\left[F_{1, s}\right] \in \bar{T}_{a+1}$ and $\alpha_{1}, \ldots, \alpha_{s} \in K$ such that

$$
\left[F_{1}\right]=\alpha_{1} \bar{\phi}_{a+1}\left[F_{1,1}\right]+\cdots+\alpha_{s} \bar{\phi}_{a+1}\left[F_{1, s}\right] .
$$

Note that $\operatorname{lcm}\left(F_{1}\right)=\operatorname{lcm}\left(F_{1, j}\right)$ for all $1 \leq j \leq s$, so $F_{1, j} \cap F_{2}=\emptyset$ for all $1 \leq j \leq s$. For all $1 \leq j \leq s$ let $\widehat{F}_{1, j}=F_{1, j} \cup F_{2}$ and assume that the elements of $\widehat{F}_{1, j}$ are ordered as the order of $G(I)$. We obtain that $[F]=\alpha_{1} \bar{\phi}_{a+b+1}\left[\widehat{F}_{1,1}\right]+\cdots+\alpha_{s} \bar{\phi}_{a+b+1}\left[\widehat{F}_{1, s}\right]$, which contradicts to the fact that $[F] \notin \operatorname{Im} \bar{\phi}_{a+b+1}$. Similarly, $\left[F_{2}\right] \notin \operatorname{Im} \bar{\phi}_{b+1}$.

It follows that

$$
t_{a+b}=\operatorname{deg}(\operatorname{lcm}(F)) \leq \operatorname{deg}\left(\operatorname{lcm}\left(F_{1}\right)\right)+\operatorname{deg}\left(\operatorname{lcm}\left(F_{2}\right)\right) \leq t_{a}+t_{b} .
$$

Corollary 4.2. Let $I$ be a monomial ideal with $G(I)=\left\{m_{1}, \ldots, m_{r}\right\}$. If $\frac{\operatorname{lcm}\left(m_{1}, \ldots, m_{r}\right)}{\operatorname{lcm}\left(m_{1}, \ldots, \widehat{m}_{i}, \ldots, m_{r}\right)} \notin K$ for all $i$, where $\widehat{m}_{i}$ means that $m_{i}$ is omitted, then $t_{a+b} \leq t_{a}+t_{b}$.

Proof. Let $[F]$ be a generator of $T_{a+b}$ with $\operatorname{deg}(\operatorname{lcm}(F))=t_{a+b}$. By the assumption, it follows that $[F] \in \operatorname{ker} \bar{\phi}_{a+b}$ and $[F] \notin \operatorname{Im} \bar{\phi}_{a+b+1}$. Hence the assertion follows from Proposition 4.1 .

Example 4.3. Let $S=K[a, b, c]$ and $I$ be the ideal of $S$ which generated by $G(I)=\left\{a^{4} b c, b^{3} c^{2}, c^{5} a^{3}\right\}$. Not that

$$
\frac{\operatorname{lcm}(G(I))}{\operatorname{lcm}\left(b^{3} c^{2}, c^{5} a^{3}\right)}=a, \frac{\operatorname{lcm}(G(I))}{\operatorname{lcm}\left(a^{4} b c, c^{5} a^{3}\right)}=b^{2}, \frac{\operatorname{lcm}(G(I))}{\operatorname{lcm}\left(a^{4} b c, b^{3} c^{2}\right)}=c^{3} .
$$

So by Corollary 4.2, I satisfies the subadditivity condition. 


\section{REFERENCES}

[1] O. Fernández-Ramos, P. Gimenez, Regularity 3 in edge ideals associated to bipartite graphs, J. Algebraic Combin. 39, 919-937 (2014).

[2] J. Herzog, H. Srinivasan, A note on the subadditivity problem for maximal shifts in free resolutions, Commutative Algebra and Noncommutative Algebraic Geometry, II. MSRI Publications, vol. 68, 245-250 (2015).

[3] L. L. Avramov, A. Conca, S. Iyengar, Subadditivity of syzygies of Koszul algebras, Math. Ann. 361, 511-534 (2015).

[4] Ali Akbar Yazdan Pour, Candidates for nonzero Betti numbers of monomila ideals, Comm. Algebra, no.4, 1488-1492 (2017).

[5] S. E. Khoury, H. Srinivasan, A note on the subadditivity of syzygies. J. Algebra Appl. 1750177 (2016).

[6] A. Abedelfatah, E. Nevo, On vanishing patterns in j-strands of edge ideals, J. Algebraic Comb. 46(2), 287-295 (2017).

[7] S. Faridi, Lattice complements and the subadditivity of syzygies of simplicial forests (2016), http://arxiv.org/abs/1605.07727

[8] M. Bigdeli and J. Herzog, Betti diagrams with special shape, Homological and Computational Methods in Commutative algebra, Springer INdAM Series 20, 33-52 (2017)

Department of Mathematics, ORT Braude College, 2161002 Karmiel, Israel

E-mail address: abed@braude.ac.il 